\title{
Microscopic Congestion Detection Protocol in VANETs
}

\author{
Mushtaq Ahmad $\mathbb{D}$, ${ }^{1}$ Qingchun Chen $\mathbb{D}^{\mathrm{D}},{ }^{2}$ and Zahid Khan ${ }^{1}$ \\ ${ }^{1}$ Southwest Jiaotong University, Chengdu, Sichuan 611756, China \\ ${ }^{2}$ Guangzhou University, Guangzhou, Guangdong 510006, China \\ Correspondence should be addressed to Qingchun Chen; qcchen@swjtu.edu.cn
}

Received 9 February 2018; Revised 24 May 2018; Accepted 7 June 2018; Published 28 June 2018

Academic Editor: María Calderon

Copyright (C) 2018 Mushtaq Ahmad et al. This is an open access article distributed under the Creative Commons Attribution License, which permits unrestricted use, distribution, and reproduction in any medium, provided the original work is properly cited.

\begin{abstract}
Effective transportation status surveillance imposes critical challenges for the Intelligent Transportation System (ITS) design. In this paper, the microscopic congestion detection protocol (MCDP) is proposed to make the vehicle-to-vehicle (V2V) communication capable of monitoring vehicle density and identifying traffic jam. By introducing transportation control domain in the existing network protocol header, each vehicle can count its neighbors and estimate the time spacing among vehicles. MCDP provides an infrastructure-less solution to the estimate of vehicle density, flow, and average velocity in a microscopically manner. Moreover, the safety speed limit is introduced to make each vehicle calculate its time to cover the intervehicle distance, such that every vehicle is able to assess the transportation congestion by comparing with some predefined safety time threshold. Monte Carlo simulations of the MCDP over four typical Chinese highways are presented to compare the MCDP scheme with the existing GreenShield congestion detection scheme. In addition, real road traces generated by SUMO over NS2 are utilized to show the achieved performance in terms of throughput, end-to-end delay, and packet delivery rate (PDR) in comparison to DSR and AOMDV in IEEE 802.11p and IEEE 802.11ac scenarios. On the basis of all the results, we conclude that MCDP is an inexpensive transport congestion detection technique for Vehicular Ad Hoc Networks (VANETs).
\end{abstract}

\section{Introduction}

The ever-increasing traffic density requires more effective traffic control technique to avoid the serious traffic jam [1]. Intelligent transportation system (ITS) provides innovative transport and traffic management technique. So far, a number of research efforts have been devoted to traffic congestion detection in both infrastructure mode and infrastructure-free mode. And these protocols aim to monitor road traffic and to estimate vehicle speed, density, and the arrival time [24]. Basically, sensing devices such as induction loop detectors [5], infrared detectors [6], microwave radars [7], and video recording devices [8] can be utilized to monitor vehicles on the highway. However, the coverage of long highway with the aforementioned dedicated devices becomes too expensive to afford the huge installations of these sensor devices. Compared to the aforementioned infrastructure method, the vehicular ad hoc network (VANET) provides us a costeffective infrastructure-free technique to support a variety of ITS applications, such as safety surveillance, road monitoring, traffic flow management, and vehicle density estimation
$[9,10]$. In the framework of the VANET, each vehicle acts as a sensor node to collect information for transportation congestion estimate. However, there are several technical problems that need to be addressed.

Basically, a reasonable information exchange mechanism among moving vehicles will be the basis to realize the traffic monitoring in the VANET framework. The direct adaptation of VANETs for traffic detection looks attractive, but it has to cope with challenges like bandwidth flooding and duplication [11], delay and inaccurate traffic evaluation [12], and the reliability problems [13]. In [2], the contents oriented communication (COC) protocol was proposed for traffic congestion and accident detection. Whenever a vehicle receives a packet, it calculates the congestion and estimates speed by itself. By exchanging the calculated results, the COC offers a feasible scheme to gather real-time position for congestion detection and speed estimation. Nonetheless, too many content exchanges in the COC protocol will consume high bandwidth. Efficient congestion detection (ECODE) protocol was proposed for VANET in [14] to evaluate traffic characteristics as well. However, ECODE uses multihop 
communications and geocast principles to collect and analyze vehicle data for each road section, which will lead to high bandwidth consumption, overhead, and complexity issues. How to design a cost-effective and efficient signaling mechanism among different vehicles to realize traffic monitoring is the first motivation of our work in this paper.

Intervehicle spacing on the highway is a critical parameter to assess traffic congestion status. Given the intervehicle spacing, the human driver can make an accurate decision to cope with the emergency situation. As an alternative, the vehicle density estimation is to know the number of vehicles per unit length of road or cell; here the cell can be any area defined by the specific clustering algorithm [15-17]. The density estimation of heterogeneous traffic was performed microscopically in [18] by assuming a fixed spacing between neighboring vehicles to simplify the model and to assess the impact of density on vehicle speed. By assuming fixed spacing, the congestion detection issues were addressed in [19-22]. Obviously, the fixed spacing assumption is not true and unrealistic. The Green-Shield car-following model was adopted in [23] for traffic congestion avoidance in single lane road. Basically, Green-Shield model is based on the macroscopic parameters like traffic density, flow, and average velocity. In this paper, the limitations of the fixed spacing among vehicles become another motivation of our work in this paper. In the proposed microscopic congestion detection protocol (MCDP) for VANETs, we considered more realistic intervehicle spacing, which is modeled as an exponential distribution.

In this paper, we propose the MCDP to reduce the computational cost by eliminating those unnecessary beacon messages exchange among vehicles to enable a cost-effective congestion detection scheme. In fact, the MCDP provides an infrastructure-less V2V approach for traffic congestion surveillance and driver assistance without installation of road side unit (RSU) or distributed sensors. The proposed MCDP will disseminate only the microscopic parameters associated with congestion detection through beacon messages and process the parameters microscopically (processing at each individual vehicle) to derive the intervehicle spacing and time headway for congestion detection, as well as speed estimation [24]. On the basis of the MCDP, the vehicles on the highway are capable of calculating the number of neighbors $N_{c}$ and estimating the spacing coverage time/headway $T_{g}$. If $T_{g}$ is less than the predefined safety threshold $\tau_{0}$, the congestion is detected, and the message will be broadcast. It should be addressed that most of the existing congestion related ITS research works are some kind of physical layer or MAC layer mechanism, while the proposed MCDP protocol is a network layer technique. As a network layer mechanism, the MCDP logically divides VANET into subsections without installation of any physical component. Hence, the network layer approach is more cost-effective than the MAC/PHY layer techniques.

In order to assess the effectiveness of the proposed MCDP protocol, we consider three typical scenarios. Firstly, theoretical analysis of MCDP over selected single and multilane Chinese highways was presented to show the achieved performance. Secondly, the MCDP characteristics were assessed through temporal congestion detection and speed estimation analysis. Thirdly, selected communication capability assessment was conducted to show the achieved network performance of the MCDP. In this paper, we have conducted a comprehensive study that combines transportation and communications, and we aim to address interdependent issues. In practice, vehicles with congestion detection and speed estimation capabilities can facilitate a safer and more comfortable driving experience. Our initial experimental analysis reveals that $\mathrm{V} 2 \mathrm{~V}$ communication among vehicles helps to refine traffic flow throughput, because it reduces the driver's perception-reaction time (PRT), which allows higher speed and safer movement of vehicles on road. All the analysis in this paper confirms that the proposed MCDP provides us with an effective network layer technique to detect and manage traffic congestion, even in the multiple lanes scenarios.

The remainder of this paper is organized as follows. In Section 2, previous works related to congestion detection are briefly reviewed to highlight the challenges. In Section 3 , the MCDP and its working philosophy are presented. Analysis and simulation results are presented in Section 4 and the paper is concluded in Section 5.

\section{The State of the Art}

Traffic evaluation always remains a concentrative point for the ITS. Many existing protocols in literature are available for traffic evaluation. In this paper, we focus on the VANET based congestion detection technology. Some VANET based congestion detection and traffic evaluation technologies are summarized in Table 1.

In addition to the protocols of ECODE [14] and COC [2], the voting protocol was proposed in [25] to estimate congestion level from neighboring vehicle characteristics. Each vehicle within transmission range disseminates its own information to neighbor vehicles, and the neighbor vehicles estimate congestion by comparing the current moving speed with the maximal allowed speed in that specific zone. The receiver vehicle cumulates the neighbor speed and votes for or against the conclusion of congestion. The congestion decision depends on the majority of votes, because it is possible that on the highway some vehicles may voluntarily move slowly. More specifically, the majority voting for slow-moving implies that highway is congested at that moment. If the other surrounding vehicles are moving at a relatively higher speed, we may arrive at the conclusion that this vehicle is voluntarily driving slowly. In contrast, if a vehicle is moving slowly over a particular road segment and the surrounding vehicles are traveling at approximately the same speed, one can conclude that congestion has occurred. Each region requires relatively high traffic density to confirm congestion decision; otherwise, congestion assessment will be less accurate.

The clustered area approach was proposed in [26], wherein the geographic area on the highway is subdivided into small managerial zones. The passing time of each zone in normal traffic condition can be defined in advance. Every vehicle measures its passing time with respect to every zone and compares it with the normal coverage time. Each 
TABLE 1: Highway traffic monitoring protocols.

\begin{tabular}{lcccc}
\hline Protocol & Architecture & Transportation & Bandwidth & Accuracy \\
\hline ECODE [14] & V2V & Directed density detection & High & Optimal \\
COC [2] & V2V & Density estimation & High & Hood \\
Voting [25] & V2V & Speed detection & Medium & Low \\
Clustered Area[26] & V2V & Arrival time estimation & High & Low \\
SOTIS[27] & V2V & Vehicle Speed & Low & Good \\
IFTIS[28] & V2V & Density detection & Low & Low \\
StreetSmart[3] & V2I & Traffic Speed & & \\
\hline
\end{tabular}

vehicle on highway exchanges its entering and leaving time with each other, which would become a prediction notice of congestion for ongoing vehicles. The frequent and large exchange of entering/exit messages with high transmission power is the primary issue in clustered area approach. Selforganizing the intervehicle network (SOTIS) [27] is another specialized approach, wherein every vehicle is assumed to be provisioned with an internal database of geographic information of highway and digital map. And all ongoing vehicles are assumed to exchange their locations, speeds, and road conditions with each other periodically. In this way, the forehead vehicle is able to inform the following vehicles about the road conditions. The main issues of the SOTIS approach are that now every vehicle is assumed to have proactive information about the highway, which will be utilized for comparison later on with the reactive information.

An infrastructure-free traffic information system (IFTIS) was proposed for vehicular networks in [28], which was the first approach in the literature to monitor road condition in a segment-wise manner. The primary advantage of the IFTIS approach is that it provides a protocol to assist the driver in selecting a noncongested lane. In the proposed IFTIS protocol, the investigated road will be segmented into overlapping location-based groups. Each group has a vehicle centralized to the cell called group leader, which has information about the whole group. The traffic density of each group is evaluated by the group leader and disseminate to the intersections. IFTIS is able to evaluate traffic density in both directions. Sometimes IFTIS experiences high density from one direction but low from other direction, and this may cause long end-to-end delays and decrease the packet delivery ratio, especially if the vehicles on the road are in the opposite direction to the desired destination. Secondly, the overlapping in clusters also reduces the accuracy of traffic evaluation across the road segment.

On the basis of clustering and epidemic communication strategy, the StreetSmart aims to find dynamic patterns and report to adjacent clusters [3]. The dynamic patterns are filtered to find out an unexpected status that is forwarded to the rest of vehicles. Each vehicle summarizes gathered statistics locally and concludes the road situation. Unfortunately, the decentralized nature of StreetSmart does not prevent the flash crowding effect nor contributes to the optimized efficiency at a global scale. After a sudden change in traffic conditions, it does not update the delay information in a timely manner. The low level of accuracy is the main limitation of the StreetSmart because a number of vehicles can get involved in multiple clusters due to the overlapping cluster areas. Other congestion detection schemes for VANETs include Virtual Sink [29], V2X [11], and Lattice [4].

A comparative study of two approaches for road traffic density estimation from traffic video scenes was presented in [30]. Both the extracted microscopic parameters (i.e., individual vehicle motion parameters) and the macroscopic parameters (i.e., global motion parameters) are applied in classifiers to enable classification of light, medium, and heavy road traffic status. It is shown that a very high accuracy can be achieved by using the traffic video classification. However, this kind of traffic monitoring is dependent on the widely deployed traffic surveillance camera, which may become too expensive to afford if we consider a very large number of cameras. Moreover, an infrared camera may be needed for the surveillance at night. Therefore, infrastructure-less solution, for instance, VANETs with reasonable traffic monitoring and congestion detection capabilities, may become highly desirable for ITS.

On the basis of the VANETs, a strategy was proposed in [31] to reduce traffic congestion, wherein periodically emitted beacons of $\mathrm{V} 2 \mathrm{~V}$ communication were proposed to enable traffic flow estimate and to warn drivers about the possible traffic breakdown. The research efforts in [31] were dedicated to the VANETS-assisted traffic jam reduction mechanism. In our work, we primarily focus on how to introduce transportation control domain in the existing network protocol header, such that each vehicle can count its neighbors and estimate the time spacing among vehicles. Because every vehicle with VANET capability is able to estimate vehicle density, flow, and average velocity in a microscopical manner, and the similar driver behavior recommendation strategy in [31] can also be employed in order to eliminate the forthcoming congestion.

Unlike all the aforementioned works, in this paper, we propose a novel approach called microscopic congestion detection protocol (MCDP), which detects the highway lane congestion and disseminate the detection results in both interlane and intralane manner. As will be shown in the following discussion, MCDP provides us with an inexpensive but effective transport congestion detection technique in VANETs.

\section{The MCDP Mechanism}

By integrating microscopic properties of vehicles [33] and external parameters, such as highway type and safety distance between vehicles, we propose a novel MCDP 


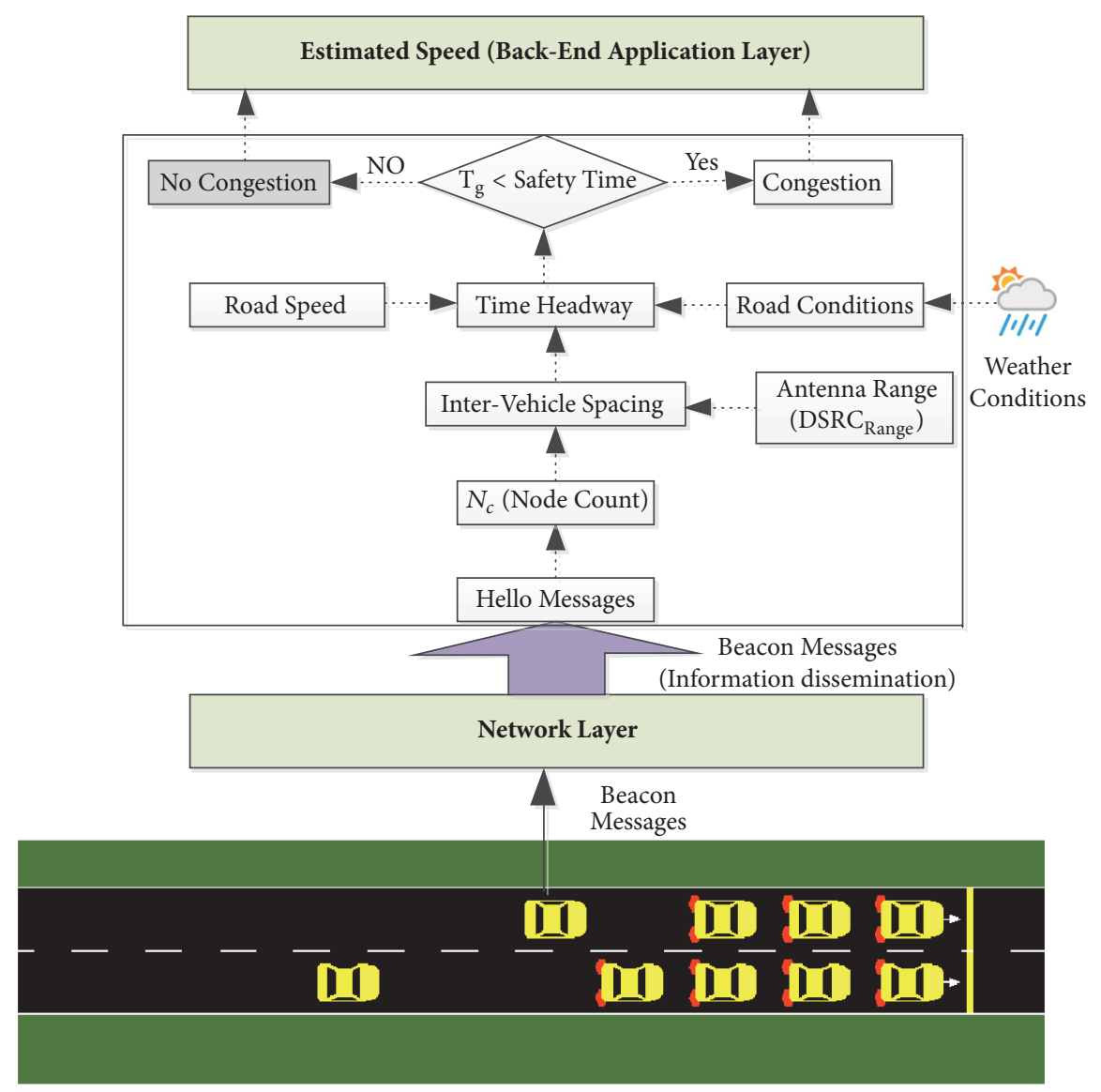

FIGURE 1: The work flow illustration of the MCDP.

to support transportation congestion detection over the existing MANETs. MCDP operates in a fully distributed infrastructure-less mode that is independent of any additional information, such as traffic data from local authorities. And MCDP is independent of the highway length. In [18], the authors considered microscopic properties for density estimation and road monitoring by assuming a fixed length intervehicle distance $d$, which is unrealistic because the fixed spacing between vehicles will affect safety regulations. Safety is an important feature of VANET, which relies on intervehicle spacing. A driver can make a reasonable decision to cope with the emergency situation only on the basis of accurate estimation of leading vehicle distance. In order to design a feasible transportation congestion detection scheme for VANETs, we should take into account the relationship between vehicle speed and interspacing. More specifically, we consider more realistic intervehicle spacing modeled as an exponential distribution. In addition, we consider macroscopic vehicle parameters that are collected microscopically for congestion detection. In MCDP, vehicles within communication range exchange macroscopic information locally, and each vehicle can thus estimate its surrounding vehicle density. The density estimation is enabled by measuring distinct neighbors and time headway (spacing coverage time) to predict upcoming congestion, as will be elaborated in detail in Section 3.1. Furthermore, every vehicle calculates its headway (spacing coverage time) and predicts upcoming congestion. The work flow of the MCDP scheme can be depicted in Figure 1, which is supposed to operating at every individual vehicle.

3.1. Information Dissemination. Information dissemination specifies the periodic exchange of beacons messages in ad hoc vehicular networks without any complicated negotiation among vehicles, which is necessary for protocol maintenance. In order to realize the transportation surveillance, it is expected to transmit the aforementioned macroscopic information to some neighboring vehicles in very short time. Many existing works, for instance [2, 14, 23, 25-28, 34-36], assume a control beacon based information dissemination mechanism as well. The information dissemination in MCDP can be subdivided into three types, namely, basic parameter exchange, speed assistance message, and interlane vehicle density.

(i) Basic Parameter Exchange: In this study, we assume that every vehicle has a GPS navigation system. On this basis, each vehicle is assumed to be able to collect vehicle current position, driving direction, speed, session time, and the topology of road networks from GIS. In the proposed MCDP framework, vehicle on the highway will periodically exchange beacon 
TABLE 2: Transportation control domain in beacon message.

\begin{tabular}{lc}
\hline Content & Description \\
\hline V_ID & Vehicle identifier \\
Session Time $T_{s}$ & Message generation time \\
Speed $S$ & Vehicle's Speed (Read from odometer) \\
Direction $d$ & Vehicle's Direction (Obtained from Navigator system) \\
Acceleration $a$ & Rate of change of Vehicle speed (Read from odometer) \\
Lane ID $L_{-} i d$ & Lane identity of Highway \\
Position $p$ & Vehicle's Position (GPS) \\
Road Type $H$ & Obtained from Transportation department \\
\hline
\end{tabular}

messages to disseminate vehicle information (vehicle ID, position, speed, lane ID, session time, and vehicle length) to all its one-hop neighbors. In this way, every vehicle can count its neighbors (vehicle density) within its DSRC communication range [37]. The details of the newly introduced transportation control domain for periodic beacon messages are given in Table 2. Each vehicle will calculate its distinct neighbor in the same Lane ID $\left(L_{i d}\right)$ and the headway/timegap between each other.

(ii) Speed Assistance Message: This message is multicast to all intralane vehicles to control the moving speed. The proposed MCDP will try to quantify the congestion level of each vehicle. As long as $T_{g}$ is lower than some predefined threshold $\tau_{0}$ (for instance, $\tau_{0}=2$ ), MCDP starts calculating the congestion level and estimating the moving speed $V_{\text {est }}$.

(iii) Interlane Vehicle Density: This message is exchanged among different highway lanes to share density level. Interlane density exchange is important for better utilization of highway lanes.

3.2. Transport Congestion Detection. Let us consider a road of length $L$, which can be subdivided into the following $n$ small road segments:

$$
\mathrm{L}=\left\{\mathrm{s}_{1}, \mathrm{~s}_{2}, \mathrm{~s}_{3}, \ldots \ldots \ldots \ldots \mathrm{s}_{\mathrm{n}}\right\}
$$

At the same time, the vehicle density on the road is assumed to follow a Poisson distribution; namely, the likelihood that $k$ vehicles are found in a space of $s$ meters can be expressed as

$$
\mathrm{P}(\mathrm{k}, \mathrm{s})=\frac{(\rho \mathrm{s})^{\mathrm{k}} \mathrm{e}^{-\rho s}}{\mathrm{k} !}, \mathrm{k} \geq 0
$$

where $\rho$ represents the vehicle density (unit: $[\mathrm{veh} / \mathrm{Km}]$ ). And the probability that there is no vehicle in the segment with length $s$ on the road is given by

$$
g(s)=P(0, s)=e^{-\rho s}
$$

The intervehicle distance is random with mean $1 / \rho_{s}$, where $\rho_{s}$ is the vehicles spatial density. The probability that there is at least one single vehicle in the segment with length $s$ can be given by

$$
\left.\mathrm{P}_{\mathrm{r}} \text { (at least one vehicle in } \mathrm{s}\right)=1-\mathrm{e}^{-\rho \mathrm{s}}
$$

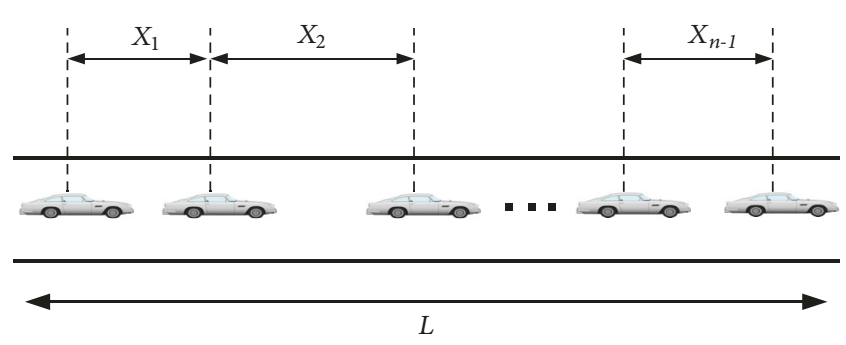

FIGURE 2: An illustrative scenario where the vehicles are randomly distributed along the road.

Let $X_{i}$ in Figure 2 represent the intervehicle distance between vehicle $i$ and vehicle $i+1$. And $X_{i}$ is independent and identically distributed (i.i.d.) with the probability density function $(p d f)$ denoted by $P_{X}(x)$ as

$$
P_{X}(x)=\lambda e^{-\lambda x}, \quad x \geq 0
$$

where $\lambda$ is the vehicle arrival rate that can be modeled as Poisson distribution and the speed $V$ assigned to the vehicles typically follows the normal distribution. If $V$ is normally distributed and intervehicle distance is exponentially distributed, the time headway $T_{g}$ is also exponentially distributed, namely,

$$
\mathrm{T}_{\mathrm{g}}(\mathrm{t})=\lambda \mathrm{e}^{-\lambda \mathrm{t}}, \quad \mathrm{t} \geq 0
$$

It can be readily derived that the time headway $t$ between two vehicles must be greater than or equal to zero since

$$
\mathrm{T}_{\mathrm{g}}(\mathrm{t} \geq 0)=\int_{0}^{\infty} \lambda \mathrm{e}^{-\lambda \mathrm{t}} \mathrm{dt}=-\mathrm{e}^{-\lambda \infty}+\mathrm{e}^{-\lambda 0}=1
$$

Congestion detection is based on the time headway $T_{g}$ among the vehicles. If the time headway $t$ is greater than the safety transportation limit $\tau_{0}$, the congestion probability will be

$$
\mathrm{T}_{\mathrm{g}}\left(\mathrm{t} \geq \tau_{0}\right)=1-\mathrm{P}_{\mathrm{T}}\left(\mathrm{t}<\tau_{0}\right)=1-\int_{0}^{\tau_{0}} \lambda \mathrm{e}^{-\lambda \mathrm{t}} \mathrm{dt}=\mathrm{e}^{-\lambda \tau_{0}}
$$

If the time headway $t$ lies between intervals of $\left(\tau_{0},(1+\delta) \tau_{0}\right)$, the congestion probability will be

$$
\begin{aligned}
\mathrm{T}_{\mathrm{g}} & {\left[\tau_{0} \leq \mathrm{t} \leq\left(\tau_{0}+\delta \tau_{0}\right)\right] } \\
& =\mathrm{P}_{\mathrm{T}}\left[\mathrm{t} \geq \tau_{0}\right]-\mathrm{P}_{\mathrm{T}}\left[\mathrm{t} \geq\left(\tau_{0}+\delta \tau_{0}\right)\right] \\
& =\mathrm{e}^{-\lambda \tau_{0}}-\mathrm{e}^{\lambda\left(\tau_{0}+\delta \tau_{0}\right)}
\end{aligned}
$$




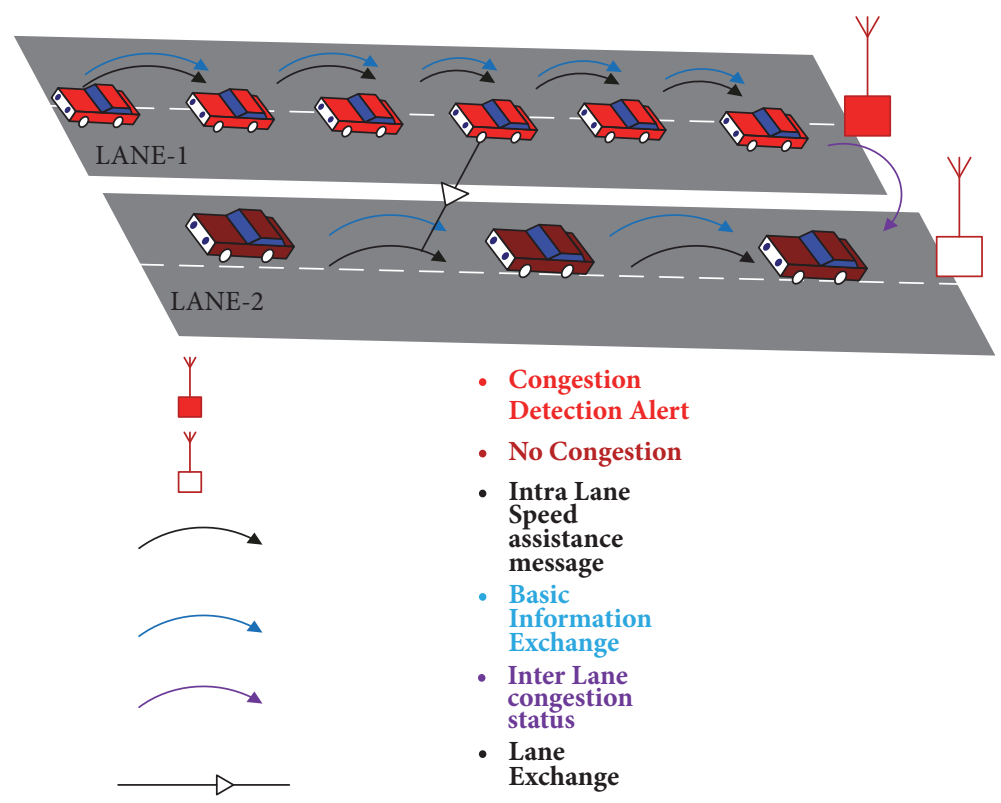

Figure 3: The MCDP enabled ITS illustration.

The vehicles collect transportation information from beacon messages and estimate traffic density. Basically, density estimation can be carried out after basic parameters exchange. Each vehicle on specific lane calculates distinct neighbors $N_{c}$ and estimates intradistance between vehicles (Spacing) on the basis of $N_{c}$. Finally, each vehicle within DSRC communication range can calculate the time-gap/Headway $T_{g}$ as

$$
\mathrm{T}_{\mathrm{g}}=\frac{1}{\text { Speed }_{\text {highway-limit }}}\left(\frac{\text { DSRC }_{\text {range }}}{\mathrm{N}_{\mathrm{c}}}-\mathrm{V}_{\text {length }}\right)
$$

where Speed $d_{\text {highway-limit }}$ corresponds to the speed limit of the corresponding highways [32], DSRC $C_{\text {range }}$ is the communication standard range [37], $N_{c}$ is the neighbor count, and $V_{\text {length }}$ is the length of the vehicle [38]. In the case of the heterogeneous traffic flow, we may consider the following mean length of vehicle:

$$
\mu_{\mathrm{V} \text {-length }}=\frac{\sum_{\mathrm{v}=1}^{\mathrm{N}_{\mathrm{c}}} \mathrm{V}_{\text {length }_{\mathrm{v}}}}{\mathrm{N}_{\mathrm{c}}}
$$

And the time headway in (10) can be rewritten as

$$
\mathrm{T}_{\mathrm{g}}=\frac{1}{\text { Speed }_{\text {highway-limit }}}\left(\frac{\text { DSRC }_{\text {range }}}{\mathrm{N}_{\mathrm{c}}}-\mu_{\mathrm{V} \text {-length }}\right)
$$

If $T_{g}$ is less than the safety transportation limit $\tau_{0}$, congestion will be detected. According to travel guide instructions, two vehicles should have at least two seconds time headway. But in certain circumstances, the safety time can be extended due to weather situations [24]. In a word, the transportation congestion detection $c$ can be summarized as follows:

$$
c= \begin{cases}1 & \text { if } T_{g}<\tau_{0} \\ 0 & \text { otherwise }\end{cases}
$$

3.3. Driver Assistance. Another notable advantage of the MCDP is its driver assistance capability. The vehicle will analyze the traffic condition based on the calculated distinct neighbors $N_{c}$ and the time headway $T_{g}$. And the appropriate speed can be estimated according to the underlying traffic status.

$$
\mathrm{V}_{\text {received }}=\frac{1}{\tau_{0}}\left(\frac{D S R C_{\text {range }}}{N_{c}}-\mu_{V \text {-length }}\right)
$$

Here $V_{\text {received }}$ is the suggested speed to the driver. The suggested speed can be multicasted intralane on the highway when $c=1$. The complete pictorial representation of MCDP is given in Figure 3, which shows the entire steps from information dissemination to congestion detection and lane alternation. Additionally, the proposed MCDP scheme can be easily extended to two-way road scenarios by separately counting the vehicles in two opposite directions on the basis of the proposed periodic beacon message exchange. Since the direction information is contained in the transportation control domain for periodic beacon messages, the two-way vehicles can be handled by two independent procedures.

\section{Experimental Study}

4.1. Analytical Assessment. Different Chinese roads are considered for analytical assessment and we also try to calculate their congestion boundaries in different scenarios. The experiments are performed with a road segment of $1 \mathrm{~km}$ having one to three lanes. The traffic is generated by Monte Carlo in Matlab R2015b [39]. To assess the performance, the proposed MCDP is compared with the Green-Shield's car-following model [23, 34]. Green-Shield's car-following model is a well-known density estimation model. Table 3 summarizes all the assumed parameters for analytical assessment. The safety time headway is considered as a performance metric. 
TABLE 3: Parameters utilized for MCDP assessment.

\begin{tabular}{lc}
\hline Parameter & Value \\
\hline Safety time/Headway & $\tau_{0}=2$ second \\
Transmission range & DSRC $_{\text {range }}=1000 \mathrm{~m}$ \\
Vehicle's length & $5 \mathrm{~m}, 10 \mathrm{~m}$ \\
Highway-type & Chinese roads [26] \\
Number of lanes & City road=1, National highway=1,Express road $=2$,Expressway=3 \\
Number of Vehicles & 2 to 70 randomly generated \\
Probability of Cars & 0.80 \\
Probability of Buses & 0.20 \\
Speed limit & $40,80,120,130 \mathrm{~km} / \mathrm{h}[32]$ \\
Lane 1 Speed & $100 \mathrm{~km} / \mathrm{h}$ \\
Lane 2 Speed & $120 \mathrm{~km} / \mathrm{h}$ \\
Lane 3 Speed & $130 \mathrm{~km} / \mathrm{h}$ \\
Simulation runs & 50 \\
Simulation time & $200 \mathrm{~s}$ \\
Propagation model & Two ray ground \\
\hline
\end{tabular}

First, spacing among vehicles is calculated under various vehicle densities and then headways are calculated under highway speed limit. Due to the microscopic feature, every individual vehicle calculates its own headway as an indicator of congestion.

The impact of vehicle density on safety time headway is shown in Figure 4. It can be observed that vehicle density and safety time have an inverse relation. The congestion boundaries of the proposed MCDP over China city road and China national highway are shown in Figures 4(a) and 4(b), where 37 and 21 vehicles are the beginning of congestion threshold, respectively. On the other hand, GreenShield model congestion threshold begins after 37 and 21 as shown in Figures 4(a) and 4(b). The exceeding of congestion boundary causes $\tau_{0}<2$ (safety time goes below than transportation limit), which further causes $c=1$ (congestion on the road). As illustrated in Figures 4(a) and 4(b), the congestion threshold of the proposed MCDP in single lane highway is less than that of the macroscopic Green-Shield's model, which implies that proposed MCDP is more sensitive to congestion detection and provides more safety traveling environment. Due to the macroscopic nature, Green-Shield model considered many irrelevant parameters (exact free flow speed and jam density), which causes communication and computational overhead. Secondly, Green-Shield model relies on expensive road monitoring devices, which can be utilized to cover specific road segment only. The congestion analysis of China express road and China expressway with multilane scenarios is shown in Figures 4(c) and 4(d). The congestion boundary of our proposed MCDP over two-lane China express road is 30 , while there are 18 vehicles in case of Green-Shield model, as shown in Figure 4(c). In twolane highway, even in the high-speed limit, the proposed MCDP model is able to accommodate a larger number of vehicles, which is desirable in order to fully utilize both lanes. Hence in the multilane highway, MCDP outperforms the reference Green-Shield model in terms of its better utilization of all available lanes. The similar observations can be found in Figure 4(d) for three-lane China expressway, where the congestion threshold of our MCDP is quite more than GreenShield. In short, by observing the numerical results of MCDP, we can conclude that our proposed MCDP protocol provides a promising approach to detect traffic congestion on the specific highway and to assist the driver.

4.2. Simulation Analysis. In this subsection, the efficiency of MCDP is analyzed under different scenarios. Two wellknown simulators such as NS2 (release 3.25) and SUMO (release 0.25.0) are used for assessment. More specifically, NS2 is used for performance evaluation of MCDP while "Sumo 0.25.0" is used for VANETs scenarios generation. Two different types of scenarios (busy congested road and freeway) with varying numbers of connections, transmission time, and simulation time are generated. To precisely simulate such a traffic monitoring system, a federated (Ubuntu 14LTS) framework is required, which combines these two simulators through generic traffic control interfaces.

4.2.1. Traffic Simulation Setup. Here SUMO is used for road traffic generation. Firstly, we designed road traffic scenario with SUMO. Roads of lengths $1 \mathrm{~km}$ and $7 \mathrm{~km}$ with two lanes are considered as an input for network simulation. The maximum speed was set to $40 \mathrm{~km} / \mathrm{h}$ for busy road and $50 \mathrm{~km} / \mathrm{h}$ for freeway scenarios, respectively. During the simulation, the red lights are turned ON to monitor all the traffic inside the fixed length of the road. The main traffic simulation parameters are summarized in Table 4.

4.2.2. Network Simulation Setup. After the generation of the mobility traces, we set up the network simulation in the NS2 framework, wherein the network simulation parameters are given in Table 5. First, busy road scenario was analyzed through MCDP by varying transmission range and simulation time. Secondly, the freeway scenario was analyzed for the same purpose with the same parameters. And we also 


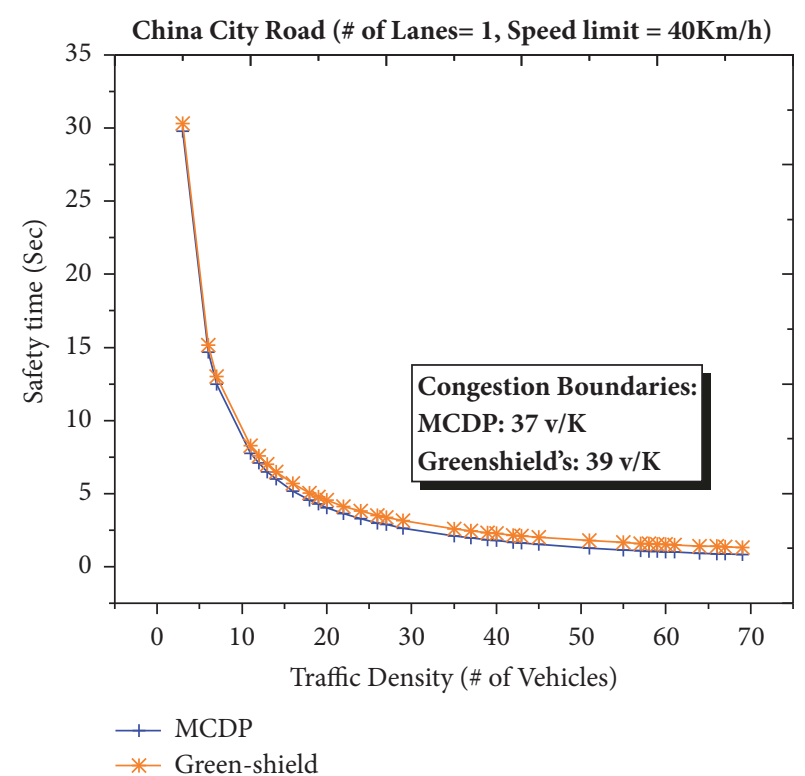

(a)

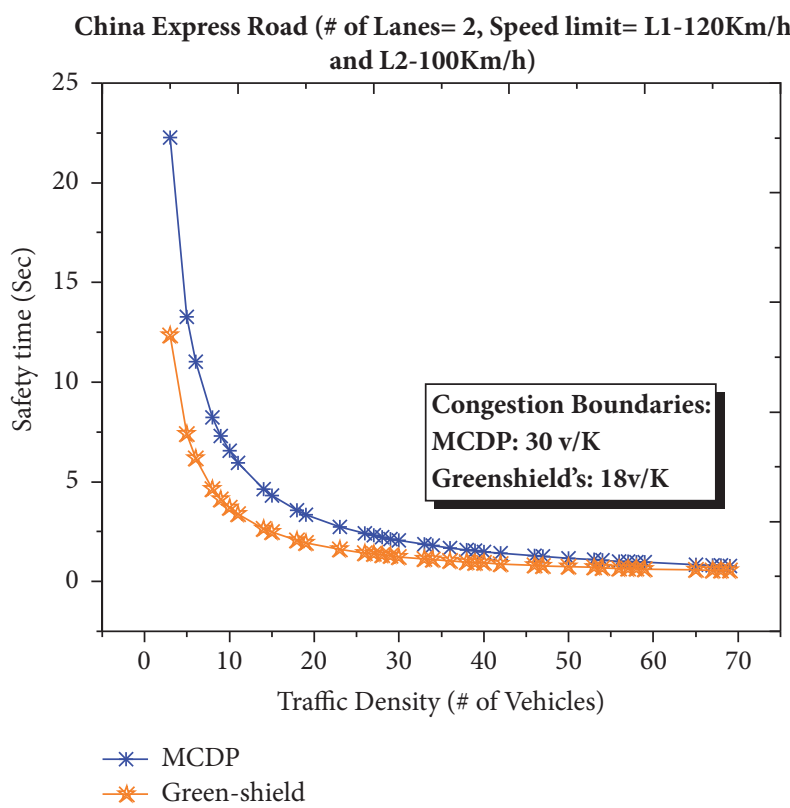

(c)

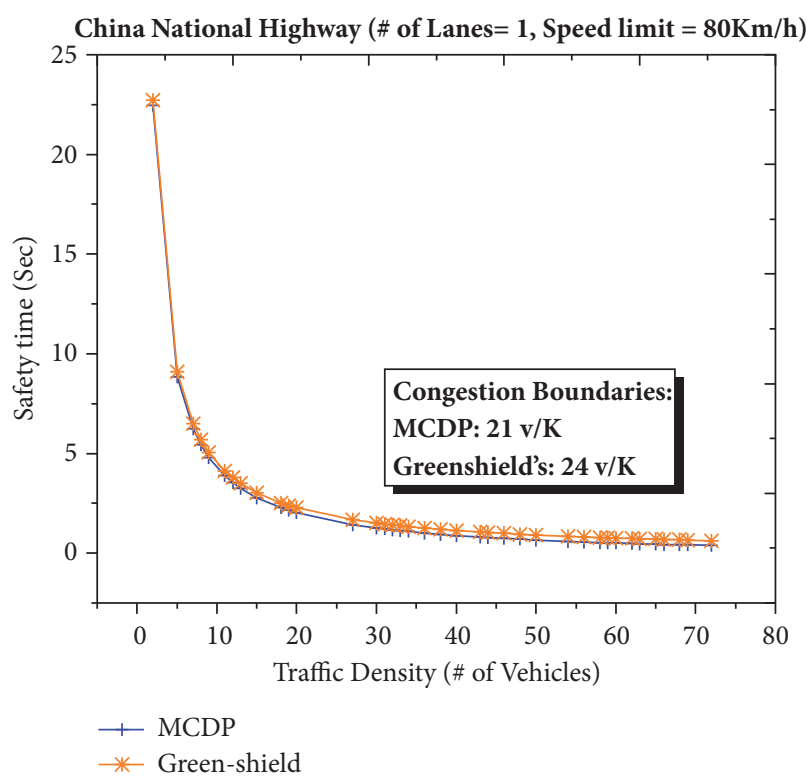

(b)

China Expressway (\# of Lanes $=3$, Speed limit $=\mathrm{L} 1-130 \mathrm{Km} / \mathrm{h}$,

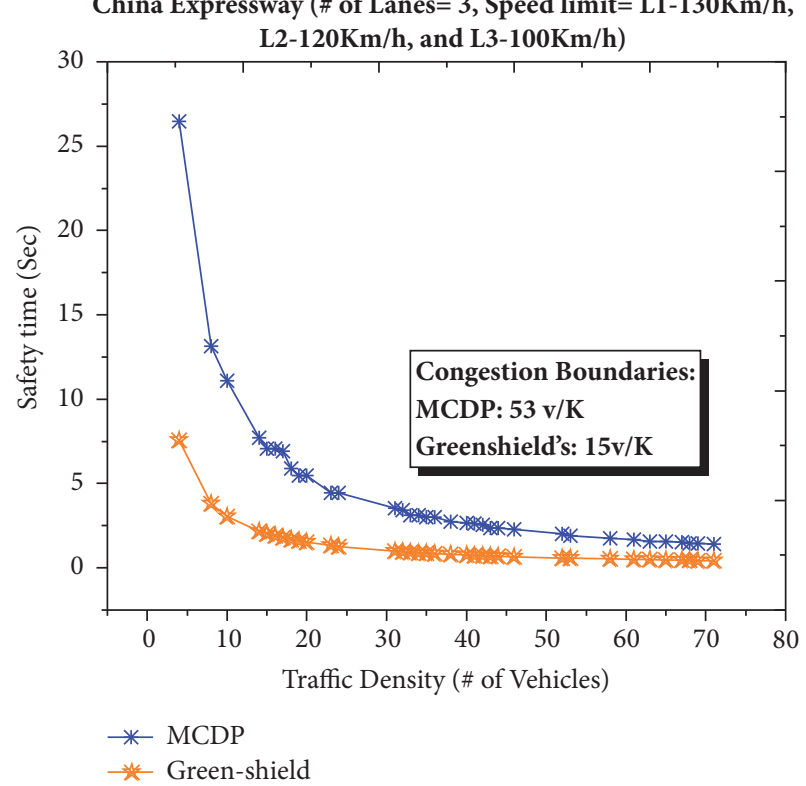

(d)

FIGURE 4: Congestion analysis in different single and multilane Chinese highways (DSRC Rang $=1000 \mathrm{~m}$, car size $=5 \mathrm{~m}$, bus size $=10 \mathrm{~m}$, and safety time threshold $=2 \mathrm{sec}$ ).

examined the performance of MCDP, DSR, and AOMDV over IEEE802.11P and IEEE802.11ac in terms of throughput, Packet delivery ratio (PDR) and end-to-end delay.

Let us briefly summarize the performance metrics utilized in the simulation analysis.

(i) Congestion Level: Congestion level is measured as a percentage of the additional travel time compared to normal traffic (or free flow situation).

(ii) Estimated Speed: Congestion is a function of the reduction in speed and vice versa. Therefore, the setting of an estimated speed that is directly related to congestion level can be used to assess the traffic congestion.

(iii) Packet Delivery Ratio (PDR): PDR is the ratio of data packets reliably delivered to the destination, i.e., $P D R=P_{r} / P_{t}$, where $P_{r}$ is the total number of received data packets and $P_{t}$ stands for the total number of transmitted data packets.

(iv) Throughput: Throughput can be utilized to assess the performance of a network by providing the average rate of successful delivery of packets towards the destination. 


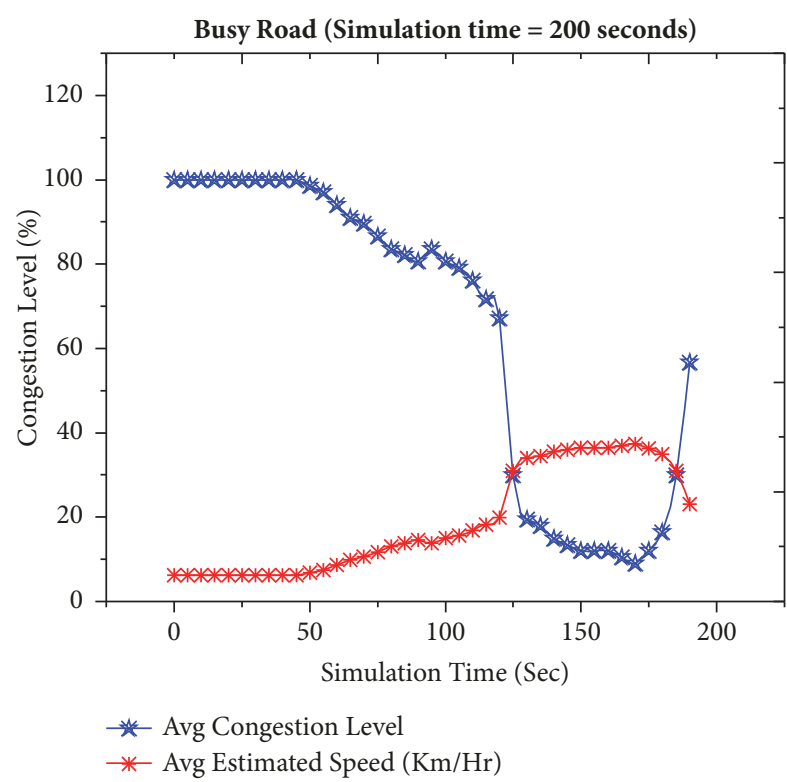

(a)

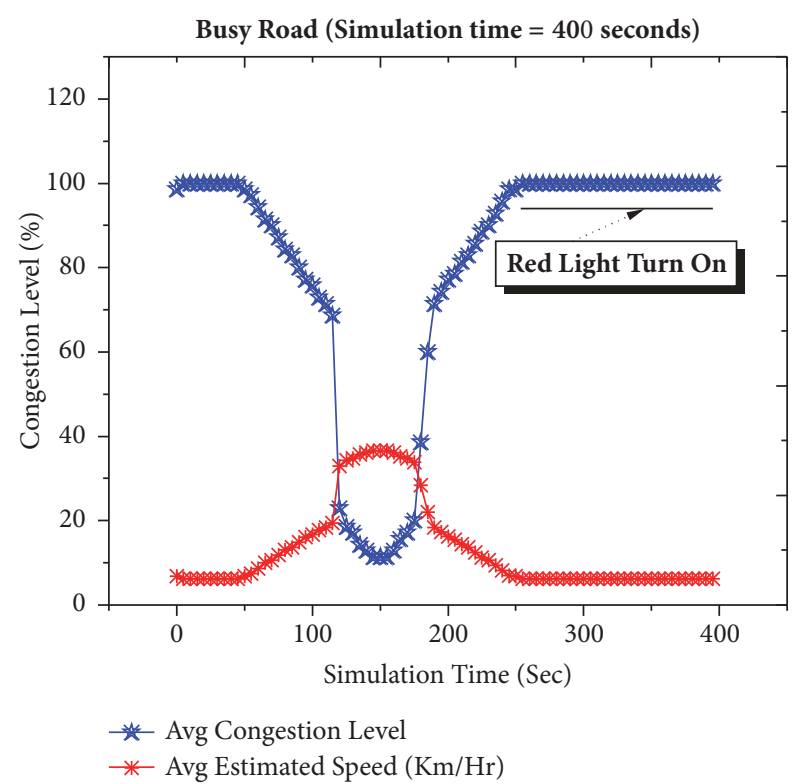

(b)

FIGURE 5: Temporal congestion detection and speed estimation in busy road, number of lanes $=2$, DSRC Rang $=500 \mathrm{~m}$, car size $=5 \mathrm{~m}$, safety time threshold $=2 \mathrm{sec}$, maximum speed $=40 \mathrm{Km} / \mathrm{H}$, total numbers of vehicles $=70$, and road length $=1 \mathrm{Km}$.

TABLE 4: Road traffic generation parameters.

\begin{tabular}{lc}
\hline Parameter & Value \\
\hline Simulator & Sumo 0.25 .0 \\
Vehicle's length & $5 \mathrm{~m}$ \\
Simulation time & $200,400,500,1000$ seconds \\
Transmission Range & $300,500,600,1000$ meters \\
Mobility Model & Busy Road and Freeway Scenarios \\
Length of the roads & 1 and 7 kilometers \\
Numbers of lanes & 2 \\
Traffic lights & 2 \\
Number of Junctions & 2 \\
Number of Nodes & Busy Road $=70$, Freeway $=50$ \\
Vehicle's Max speed & Busy Road $=40 \mathrm{Km} / \mathrm{h}$, Freeway $=50 \mathrm{Km} / \mathrm{h}$ \\
\hline
\end{tabular}

(v) End-to-End Delay: End-to-end delay is the average duration that each packet can be received by the last node in the network.

4.2.3. Congestion Detection Analysis. The proposed protocol assumes that each vehicle monitors local traffic congestion detection by analyzing the received beacon messages with detailed information from other vehicles, while each vehicle shares information about its position, direction, speed, and so on. To see the overall performance of the MCDP protocol, the average temporal congestion level and average estimated speed of all vehicles under two different traffic scenarios are shown in Figures 5 and 6, respectively. It can be observed that when the safety time goes below the safety time threshold value $\tau_{0}$, the congestion level starts to increase and the estimated speed starts to decrease. The results show that when red lights are turned $\mathrm{ON}$, the congestion is at its peak level and the estimated speed tends to be negligible. The average estimated speed to the drivers in varying traffic densities is shown as well in both Figures 5 and 6 . In all traffic and simulation setups, the following relationships among safety time $t_{\text {safe }}$, congestion level $C_{\text {level }}$, and estimated speed $S_{\text {est }}$ are concluded:

$$
\begin{gathered}
\mathrm{C}_{\text {level }} \propto \frac{1}{\mathrm{t}_{\text {safe }}} \\
\mathrm{S}_{\text {est }} \propto \mathrm{t}_{\text {safe }}
\end{gathered}
$$

In [40], the road gets into the congested state if the vehicle travel time exceeds the normal travel time at free flow. In order to do that, the scheme requires that each section of the road should be under surveillance all the time; meanwhile all the vehicles need to report their traversal time over each section to a centralized entity. In MCDP, the congestion detection can be realized without such an infrastructure. It should be addressed that the speed of a vehicle is directly affected by the level of traffic congestion in its surroundings, and it is reasonable to use it for traffic congestion detection and speed estimation. Additionally, MCDP quantifies the level of congestion locally from the information in the beacon message, which is remarkably important for delay-sensitive applications.

We illustrate the impact of transmission range (TR) for the MCDP in Figure 7. Here three different TRs of $300 \mathrm{~m}, 600 \mathrm{~m}$, and $1000 \mathrm{~m}$ are considered and the congestion detection and speed estimation technique are confined to the vehicle's visibility based on its transmission (DSRC) range. It can be noted that congestion detection level gets decreased 
TABLE 5: Network simulation parameters.

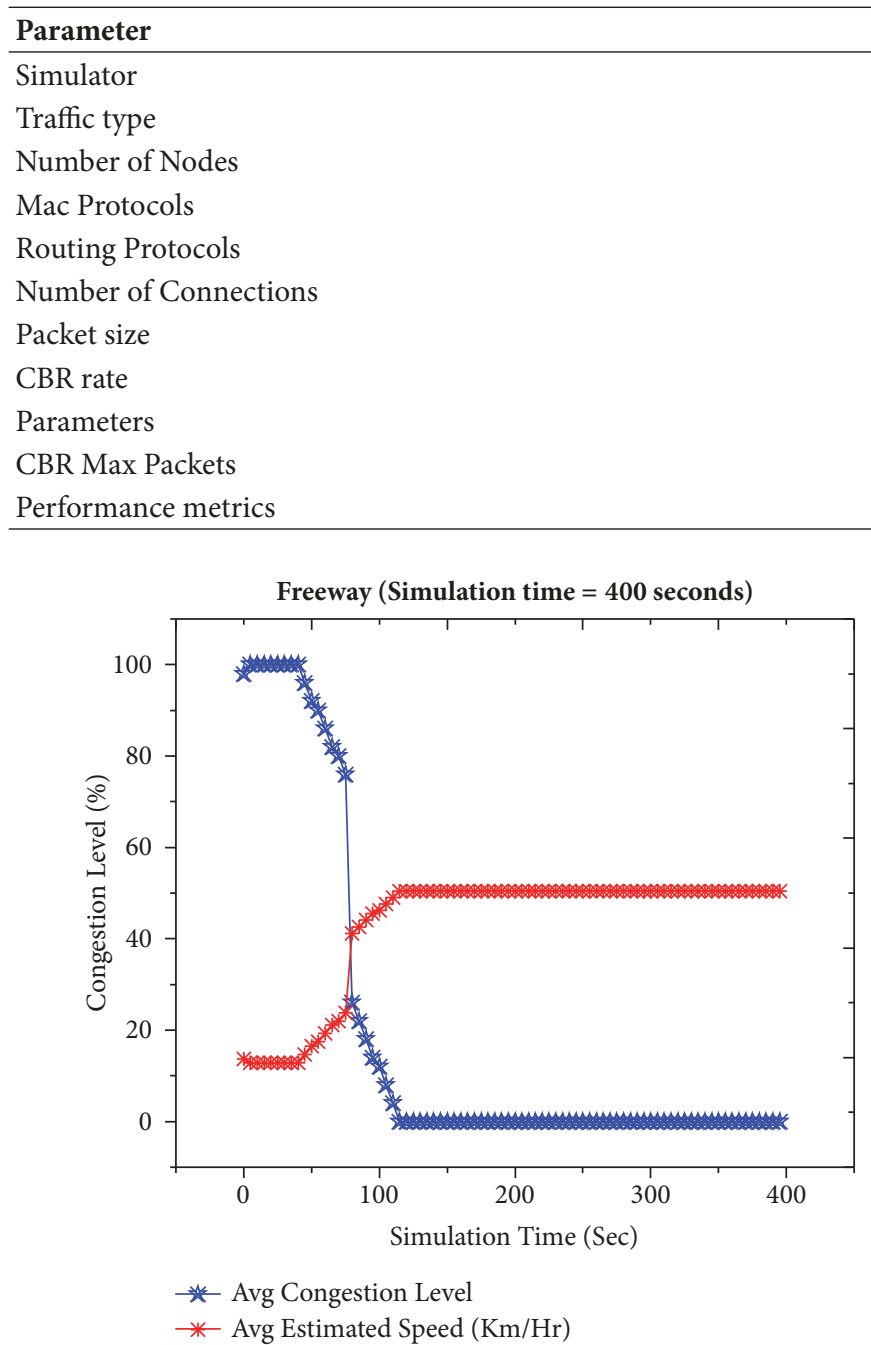

(a)

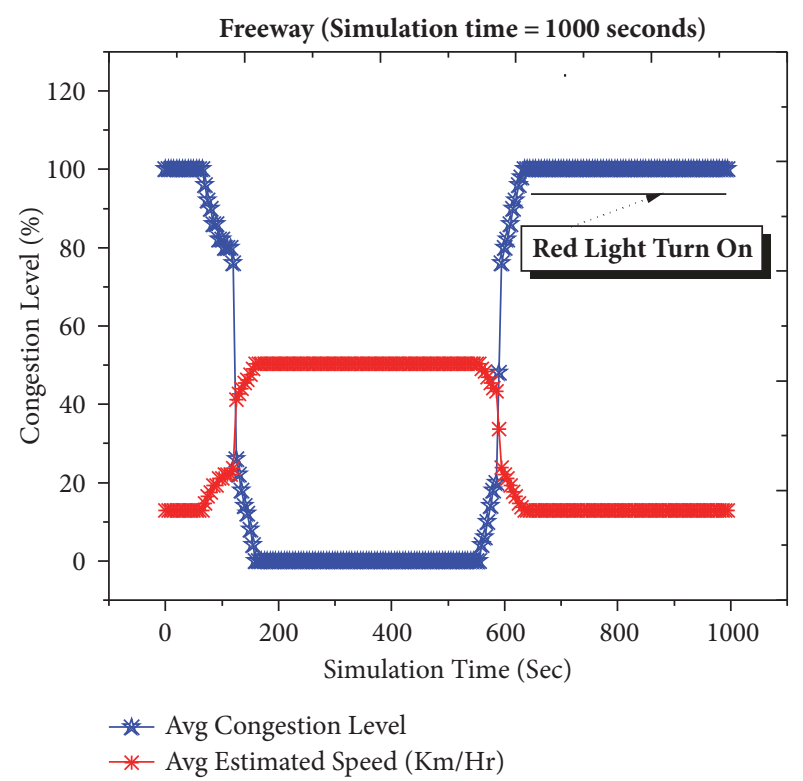

(b)

FIGURE 6: Temporal congestion detection and speed estimation in freeway, number of lanes $=2$, DSRC Rang $=500 \mathrm{~m}$, car size $=5 \mathrm{~m}$, safety time threshold $=2 \mathrm{sec}$, maximum speed $=50 \mathrm{Km} / \mathrm{h}$, total numbers of vehicles $=50$, and road length $=7 \mathrm{Km}$.

with the increase in transmission range. As illustrated in Figure $7(\mathrm{a})$, if $1000 \mathrm{~m}$ transmission range is assumed, even a traffic congestion happens, it may be ignored by the average congestion level detection assessment scheme. This suggests us that a reasonable setup of TR is important to achieve the reasonable congestion detection in the MCDP framework. A very small TR setup may make the system too sensitive to the traffic variation on road, while a too large TR will ignore the real traffic congestion. A reasonable TR setup can realize the proper tradeoff.

4.2.4. Communication Performance Analysis. MCDP is a fullfledge protocol, which is easily implementable and executable to any sort of environment, but the performance of routing protocol also depends on how better the routing takes place in the network. Here we focus on the achieved throughput, end-to-end delay, and PDR performance to assess the routing capability and efficiency of MCDP. The communication performance simulation results of MCDP, DSR, and AOMDV protocols are shown in Figure 8 with a different numbers of UDP connections over IEEE802.11p and IEEE802.11ac, respectively. From Figure 8 it can be easily concluded that, with the increase in a number of connections, the throughput of all routing protocols gets increased. While the achieved throughput of both MCDP and AOMDV protocols is almost the same for all connection numbers. In fact, due to multipath nature, their throughout is better than that of the DSR protocol. The end-to-end delay is illustrated in Figure 9 by considering UDP connections over IEEE802.11p and IEEE802.11ac. It is shown that IEEE802.11p always outperforms IEEE802.11ac in all cases, which complies with the design objective of IEEE802.11p to support the communication link between the vehicles that might exist only for a short amount of time. At the same time, it 


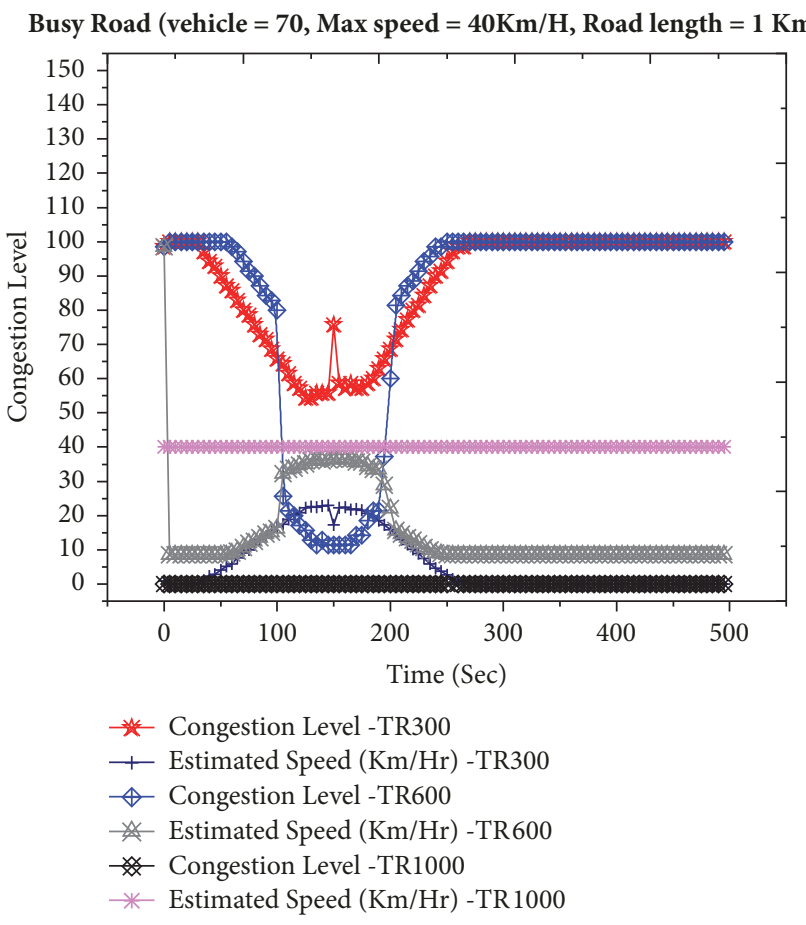

(a)

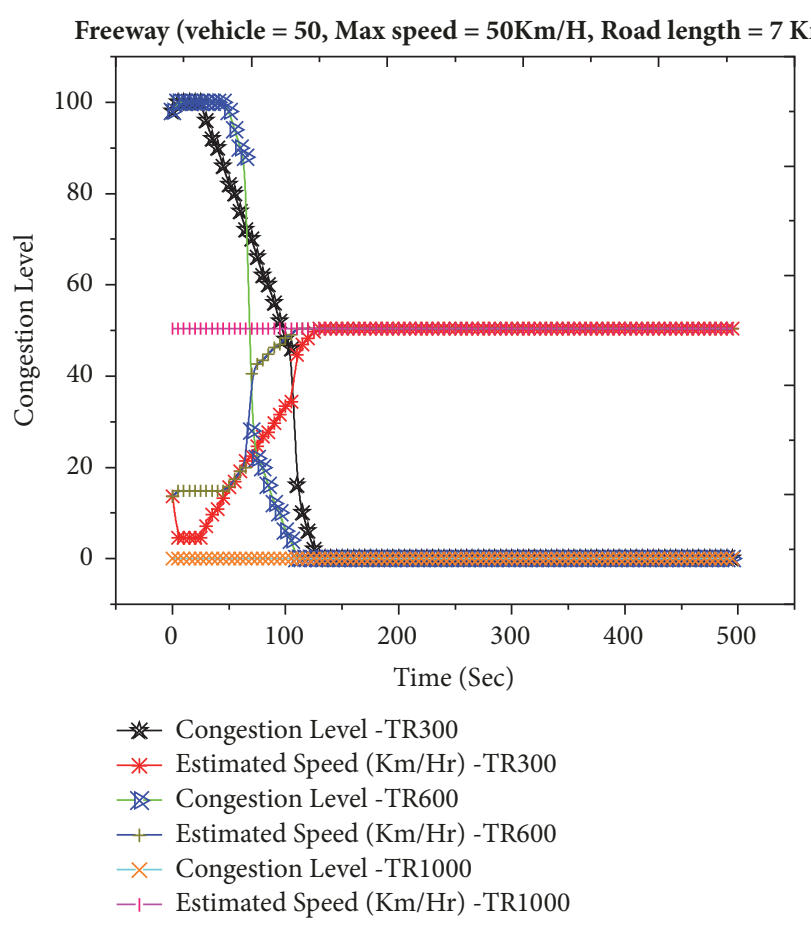

(b)

FIGURE 7: Temporal congestion detection and speed estimation using different transmission ranges, number of lanes $=2$, car size $=5 \mathrm{~m}$, safety time threshold $=2 \mathrm{sec}$, and simulation time $=500$ seconds.

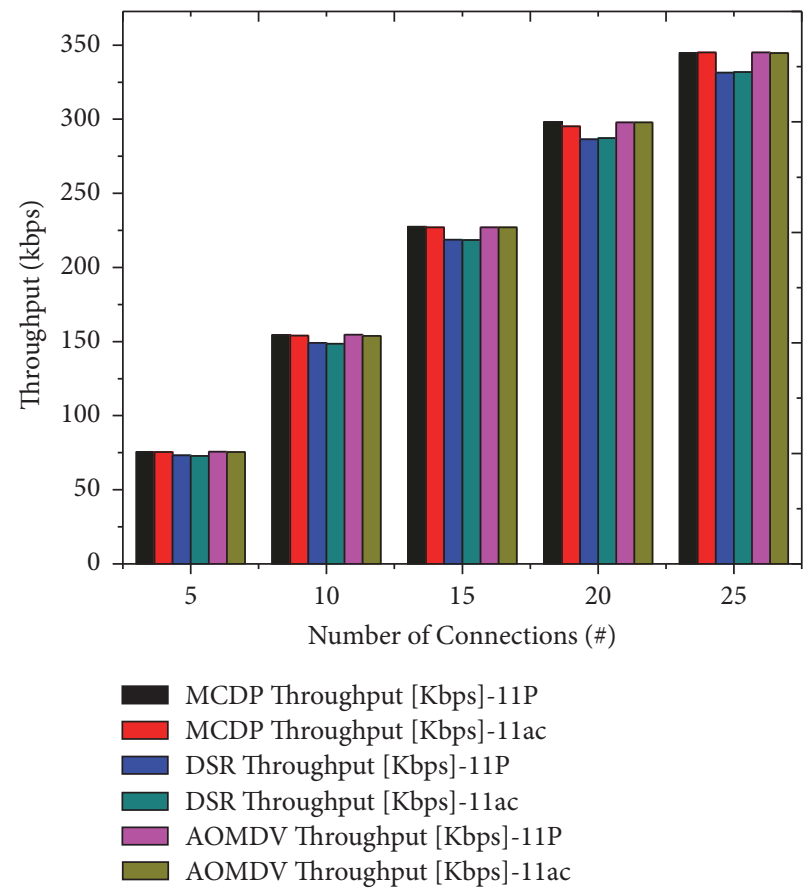

Figure 8: Throughput comparison of MCDP, DSR, and AOMDV protocols over 802.11P and 802.11ac with different numbers of connections.

can be observed that the proposed MCDP protocol can achieve a comparable good end-to-end delay and throughput performance while having congestion detection and speed estimation feature.
Finally, the PDR performances of MCDP, DSR, and AOMDV are illustrated in Figure 10 for a different number of connections. It is shown that the proposed MCDP protocol can realize comparable PDR performance as the AOMDV 

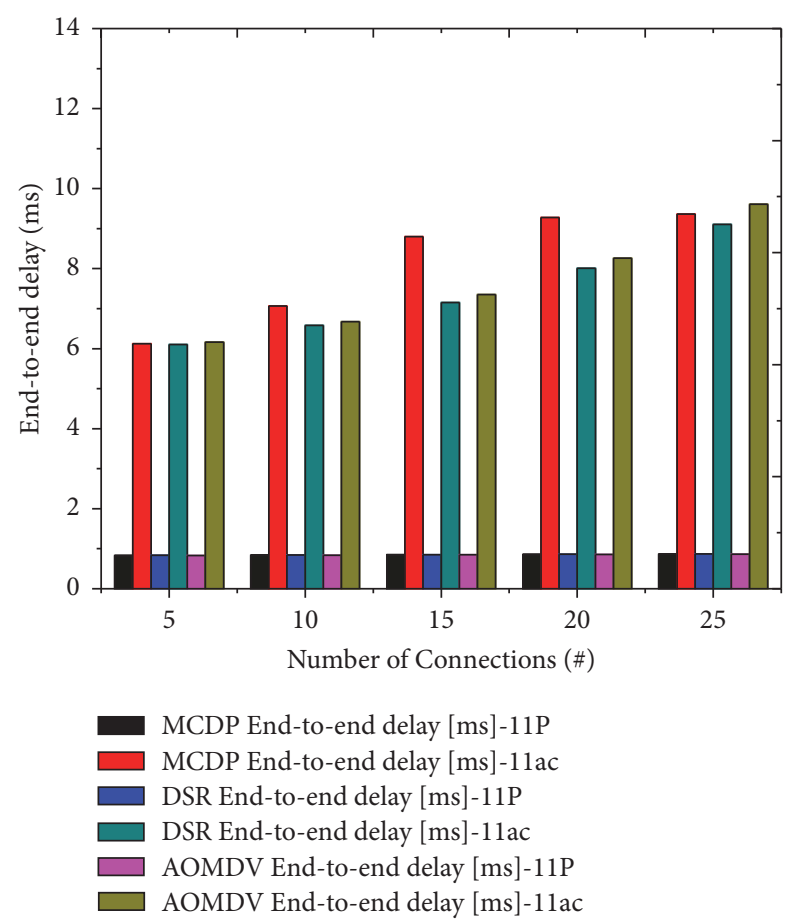

FIGURE 9: End-to-end delay comparison of MCDP, DSR, and AOMDV protocols over 802.11P and 802.11ac with different numbers of connections.

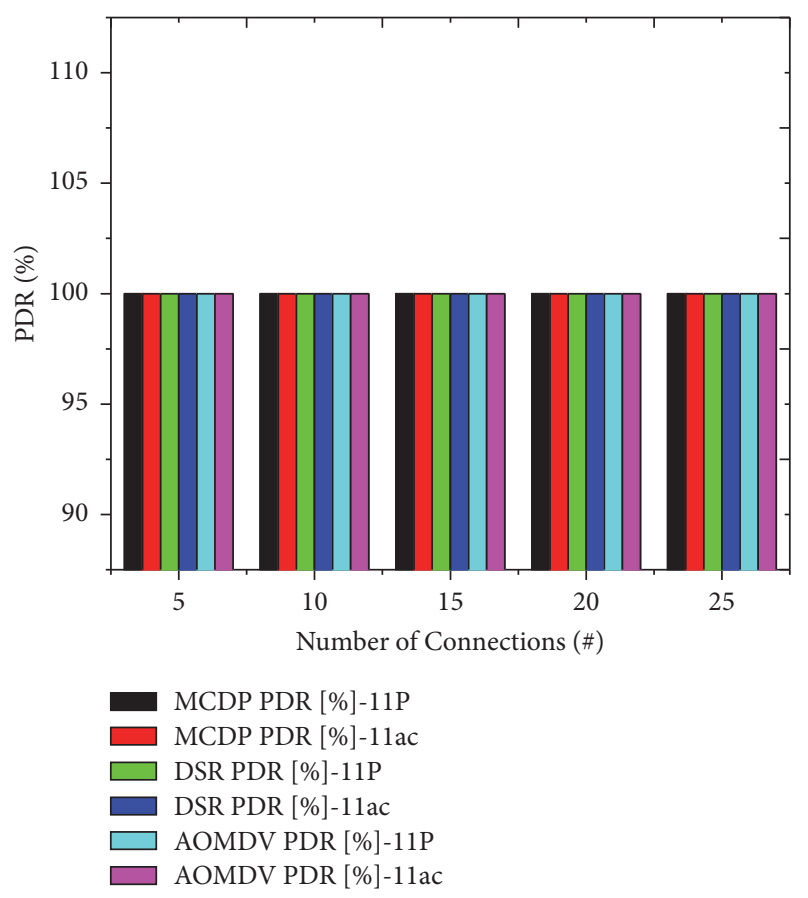

FIGURE 10: PDR comparison of MCDP, DSR, and AOMDV protocols over 802.11P and 802.11ac with different numbers of connections.

and DSR protocols in a different number of connections. Meanwhile, it can also be noted that when there is sufficient connectivity, we can almost guarantee successful packet delivery for the aforementioned three protocols.

\section{Conclusions}

VANETs technology plays an important role in safety transportation. Microscopic congestion detection protocol (MCDP) is an interesting application of VANET technology to identify road congestion. At the same time, MCDP provides us with a new approach to estimate vehicles density and to assist driver assistance. In a word, MCDP is an inexpensive approach, which integrates basic microscopic vehicle properties with some external road safety parameters to accurately monitor road status. Moreover, MCDP is quite simple protocol and it works both in a single lane and in multilane highway. An appropriate speed suggestion to the driver at every moment is also a potential application of MCDP, which will be left for future investigation.

\section{Data Availability}

The data used to support the findings of this study are available from the corresponding author upon request.

\section{Conflicts of Interest}

The authors declare that they have no conflicts of interest.

\section{Acknowledgments}

This work was partly supported by the National Natural Science Foundation of China (NSFC) under Grant no. 61771406.

\section{References}

[1] The world's worst traffic jams. http://content.time.com/time/ world/article/0,8599,1733872,00.html

[2] J. Fukumoto, N. Sirokane, Y. Ishikawa, T. Wada, K. Ohtsuki, and H. Okada, "Analytic method for real-time traffic problems by using Contents Oriented Communications in VANET," in Proceedings of the 2007 th International Conference on ITS Telecommunications, pp. 1-6, Sophia Antipolis, France, June 2007.

[3] S. Dornbush and A. Joshi, "Streetsmart traffic: discovering and disseminating automobile congestion using vanet's," in Proceedings of the 65th IEEE Vehicular Technology Conference (VTC '07), pp. 11-15, Dublin, Republic of Ireland, April 2007.

[4] S. Inoue, K. Shozaki, and Y. Kakuda, "An automobile control method for alleviation of traffic congestions using inter-vehicle ad hoc communication in lattice-like roads," in Proceedings of the IEEE Globecom Workshops, pp. 1-6, IEEE, Washington, DC, USA, November 2007.

[5] R. Morla, Vision of Congestion-Free Road Traffic and Cooperating Objects, citeulike: 3806024, 2005.

[6] S. Roy, R. Sen, S. Kulkarni, P. Kulkarni, B. Raman, and L. K. Singh, "WirelessAcrossRoad: RF based road traffic congestion detection," in Proceedings of the 2011 3rd International Conference on Communication Systems and Networks, COMSNETS 2011, pp. 1-6, i, January 2011.

[7] D. Huang, S. Shere, and S. Ahn, "Dynamic highway congestion detection and prediction based on shock waves, dynamic highway congestion detection and prediction based on shock waves," in Proceedings of the ACM VANET '10, pp. 11-20, 2010. 
[8] P. Pongpaibool, P. Tangamchit, and K. Noodwong, "Evaluation of road traffic congestion using fuzzy techniques," in Proceedings of the IEEE Region 10 Conference, TENCON 2007, pp. 1-4, November 2007.

[9] E. Hossain, G. Chow, V. C. M. Leung et al., "Vehicular telematics over heterogeneous wireless networks: a survey," Computer Communications, vol. 33, no. 7, pp. 775-793, 2010.

[10] M. Seredynski and P. Bouvry, "A survey of vehicular-based cooperative traffic information systems," in Proceedings of the 14th IEEE International Intelligent Transportation Systems Conference, ITSC 2011, pp. 163-168, October 2011.

[11] B. Schunemann, J. Wedel, and I. Radusch, "V2X-based traffic congestion recognition and avoidance," Tamkang Journal of Science and Engineering, vol. 13, no. 1, pp. 63-709, 2010.

[12] T. Zhong, B. Xu, and O. Wolfson, "Disseminating real-time traffic information in vehicular ad-hoc networks," in Proceedings of the 2008 IEEE Intelligent Vehicles Symposium, IV, pp. 1056-1061, June 2008.

[13] G. Korkmaz, F. Özgüner, E. Ekici, and Ü. Özgüner, "Urban multi-hop broadcast protocol for inter-vehicle communication systems," in Proceedings of the First ACM International Workshop on Vehicular Ad Hoc Networks, Held in Conjunction with MOBICOM 2004, pp. 76-85, October 2004.

[14] M. Bani Younes and A. Boukerche, "A performance evaluation of an efficient traffic congestion detection protocol (ECODE) for intelligent transportation systems," Ad Hoc Networks, vol. 24, pp. 317-336, 2015.

[15] B. S. Kerner, Introduction to Modern Traffic Flow Theory and Control, Springer, Berlin, Germany, 2009.

[16] M. Jerbi, S.-M. Senouci, T. Rasheed, and Y. Ghamri-Doudane, "Towards efficient geographic routing in urban vehicular networks," IEEE Transactions on Vehicular Technology, vol. 58, no. 9, pp. 5048-5059, 2009.

[17] S. Bilal, S. Madani, and I. Khan, "Enhanced junction selection mechanism for routing protocol in VANETs," International Arab Journal of Information Technolog, vol. 8, no. 4, pp. 422429, 2011.

[18] T. Umer, Z. Ding, B. Honary, and H. Ahmad, "Implementation of microscopic parameters for density estimation of heterogeneous traffic flow for VANET," in Proceedings of the 2010 7th International Symposium on Communication Systems, Networks and Digital Signal Processing, CSNDSP 2010, pp. 66-70, July 2010.

[19] J. Ploeg, E. Semsar-Kazerooni, G. Lijster, N. Van De Wouw, and H. Nijmeijer, "Graceful degradation of CACC performance subject to unreliable wireless communication," in Proceedings of the 2013 16th International IEEE Conference on Intelligent Transportation Systems: Intelligent Transportation Systems for All Modes, ITSC 2013, pp. 1210-1216, October 2013.

[20] P. Fernandes and U. Nunes, "Platooning with IVC-enabled autonomous vehicles: Strategies to mitigate communication delays, improve safety and traffic flow," IEEE Transactions on Intelligent Transportation Systems, vol. 13, no. 1, pp. 91-106, 2012.

[21] J. Zhou and H. Peng, "Range policy of adaptive cruise control vehicles for improved flow stability and string stability," IEEE Transactions on Intelligent Transportation Systems, vol. 6, no. 2, pp. 229-237, 2005.

[22] P. Seiler, A. Pant, and K. Hedrick, "Disturbance propagation in vehicle strings," Institute of Electrical and Electronics Engineers Transactions on Automatic Control, vol. 49, no. 10, pp. 1835-1841, 2004.
[23] M. Kimura, Y. Taoda, Y. Kakuda, S. Inoue, and T. Dohi, "A novel method based on VANET for alleviating traffic congestion in urban transportations," in Proceedings of the 2013 IEEE Eleventh International Symposium on Autonomous Decentralized Systems (ISADS), pp. 1-7, Mexico City, Mexico, March 2013.

[24] http://www.sdt.com.au/safedrive-directory-FOLLOWINGDISTANCE.htm.

[25] F. M. Padron, Traffic Congestion Detection Using VANET [M.S.C.S. Thesis], Florida Atlantic University, 2009.

[26] N. Shibata, T. Terauchi, T. Kitani, K. Yasumoto, M. Ito, and T. Higashino, "A method for sharing traffic jam information using inter-vehicle communication," in Proceedings of the 2006 3 rd Annual International Conference on Mobile and Ubiquitous Systems: Networking and Services, MobiQuitous, pp. 1-7, July 2006.

[27] L. Wischhof, A. Ebner, and H. Rohling, "Information dissemination in self-organizing intervehicle networks," IEEE Transactions on Intelligent Transportation Systems, vol. 6, no. 1, pp. 90-101, 2005.

[28] M. Jerbi, S.-M. Senouci, R. Meraihi, and Y. Ghamri-Doudane, "An improved vehicular ad hoc routing protocol for city environments," in Proceedings of the 2007 IEEE International Conference on Communications, ICC'07, pp. 3972-3979, June 2007.

[29] L.-D. Chou, D. Chunhu Li, and H.-W. Chao, "Mitigate traffic congestion with virtual data sink based information dissemination in Intelligent Transportation System," in Proceedings of the 3rd International Conference on Ubiquitous and Future Networks, ICUFN 2011, pp. 37-42, June 2011.

[30] O. Asmaa, K. Mokhtar, and O. Abdelaziz, "Road traffic density estimation using microscopic and macroscopic parameters," Image and Vision Computing, vol. 31, no. 11, pp. 887-894, 2013.

[31] F. Knorr, D. Baselt, M. Schreckenberg, and M. Mauve, "Reducing traffic jams via VANETs," IEEE Transactions on Vehicular Technology, vol. 61, no. 8, pp. 3490-3498, 2012.

[32] http://wikitravel.org/en/Driving_in_China.

[33] M. H. Eiza and Q. Ni, "An evolving graph-based reliable routing scheme for VANETs," IEEE Transactions on Vehicular Technology, vol. 62, no. 4, pp. 1493-1504, 2013.

[34] M. Milojevic and V. Rakocevic, "Distributed road traffic congestion quantification using cooperative VANETs," in Proceedings of the 2014 13th Annual Mediterranean Ad Hoc Networking Workshop, MED-HOC-NET 2014, pp. 203-210, June 2014.

[35] G. B. Araujo, M. M. Queiroz, F. d. Duarte-Figueiredo, A. I. Tostes, and A. A. Loureiro, "CARTIM: A proposal toward identification and minimization of vehicular traffic congestion for VANET," in Proceedings of the 2014 IEEE Symposium on Computers and Communication (ISCC), pp. 1-6, Funchal, Madeira, Portugal, June 2014.

[36] F. Terroso-Sáenz, M. Valdés-Vela, C. Sotomayor-Martínez, R. Toledo-Moreo, and A. F. Gómez-Skarmeta, "A cooperative approach to traffic congestion detection with Complex Event processing and VANET," IEEE Transactions on Intelligent Transportation Systems, vol. 13, no. 2, pp. 914-929, 2012.

[37] X. Ma, X. Chen, and H. H. Refai, "Performance and reliability of DSRC vehicular safety communication: a formal analysis," EURASIP Journal on Wireless Communications and Networking, vol. 1, 2009.

[38] Wikipedia, "Car Dimension" [EB/OL], 2015, http://en.wikipedia.org/wiki/car_dimension. 
[39] P. Brémaud, Markov Chains: Gibbs Fields, Monte Carlo Simulation, and Queues, vol. 31, Springer Science \& Business Media, 2013.

[40] G. Marfia and M. Roccetti, "Vehicular congestion detection and short-term forecasting: a new model with results," IEEE Transactions on Vehicular Technology, vol. 60, no. 7, pp. 29362948, 2011. 


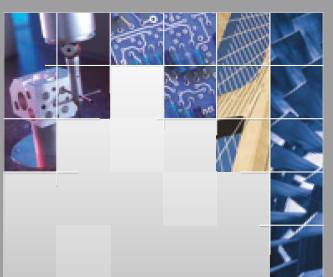

\section{Enfincering}
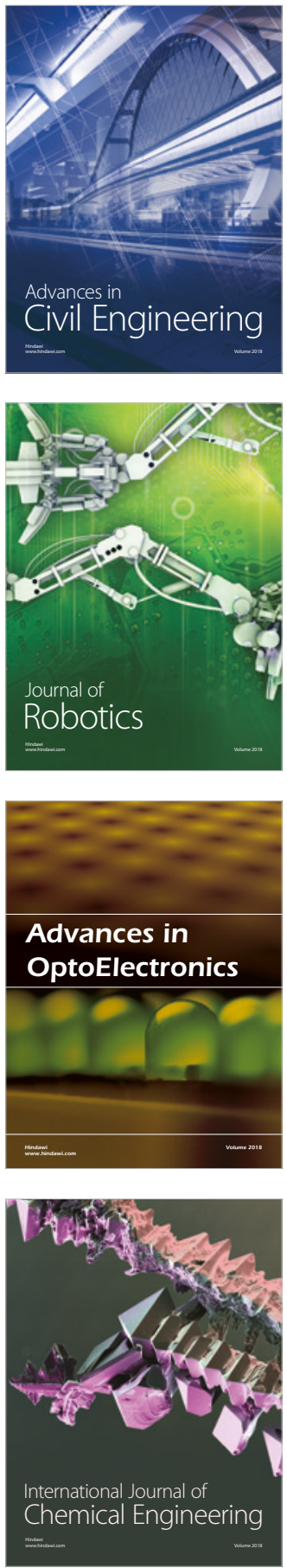

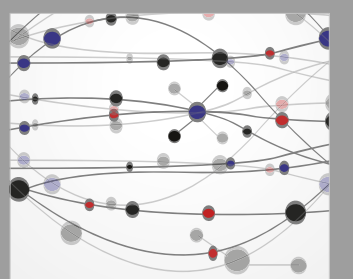

\section{Rotating \\ Machinery}

The Scientific World Journal

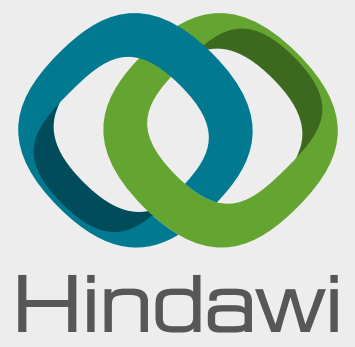

Submit your manuscripts at

www.hindawi.com
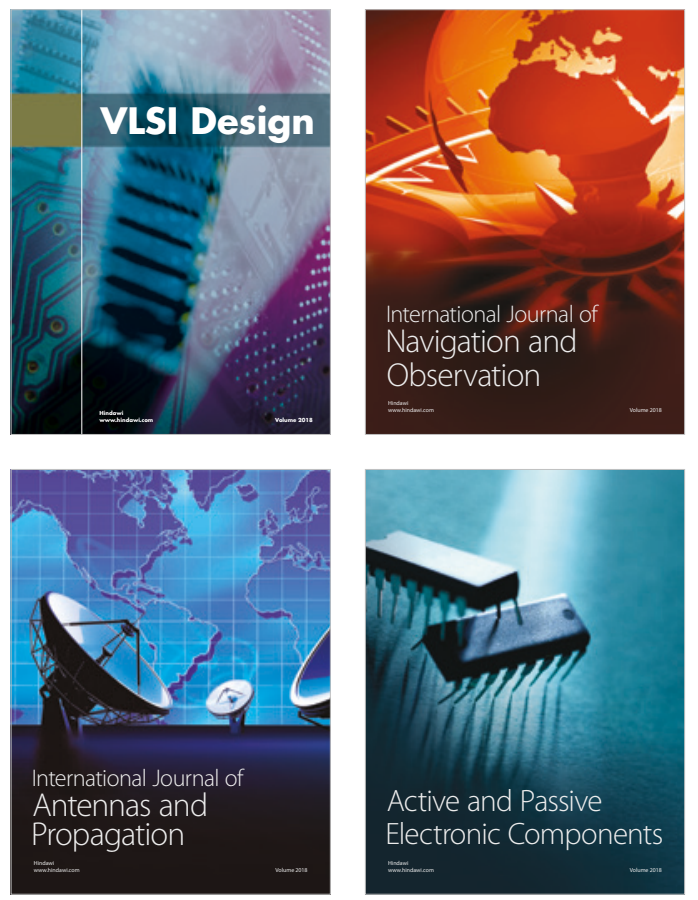
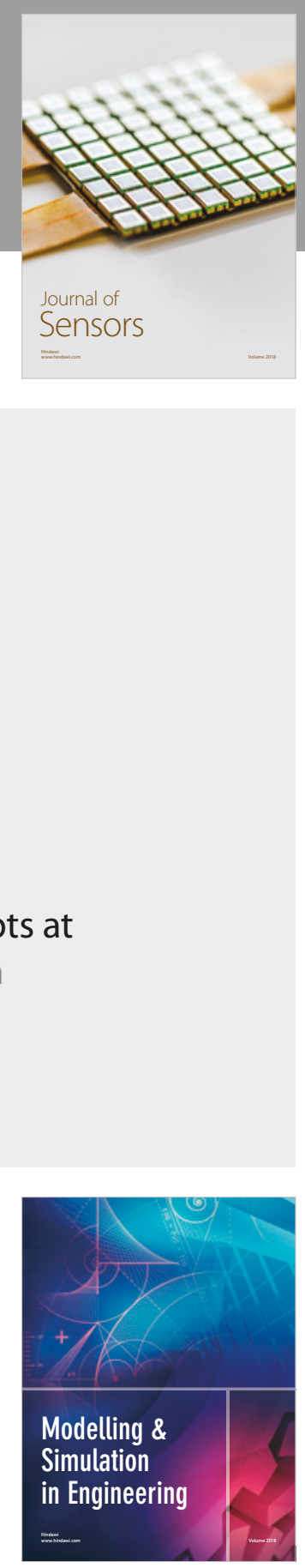

\section{Advances \\ Multimedia}
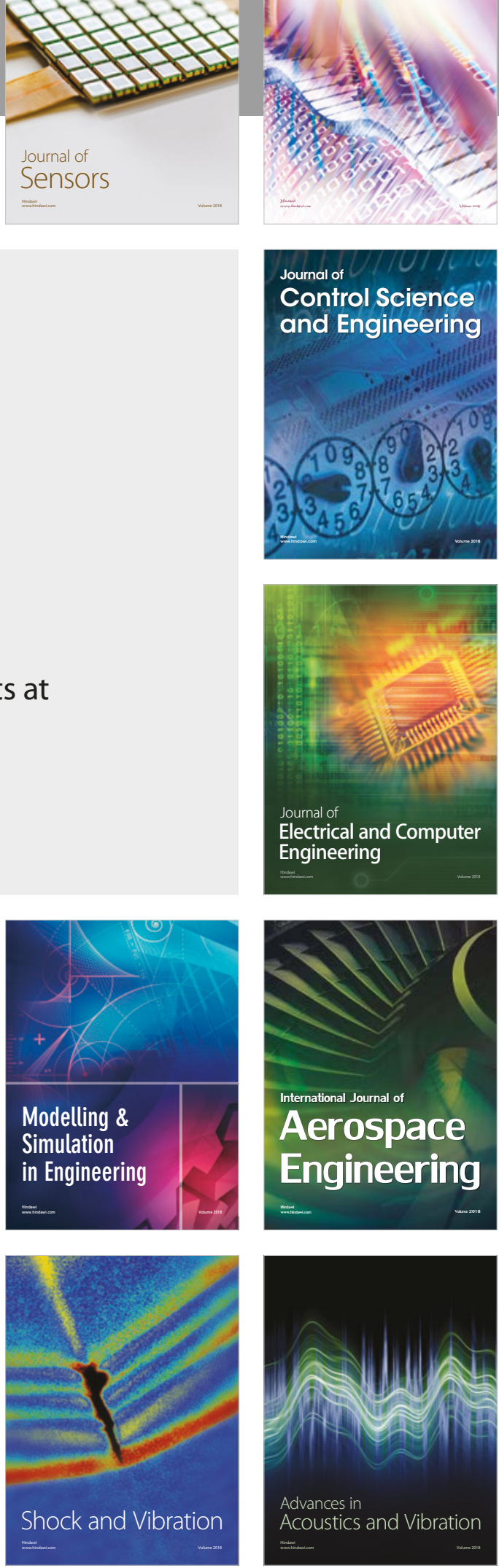